\title{
CREB down-regulation in the laterodorsal thalamic nucleus deteriorates memory consolidation in rats
}

\author{
Wenxi Xiang, Tingting Li, Tianhang Gao, and Bin Wang \\ Department of Anatomy, College of Basic Medicine, Dalian Medical University, Dalian 116044, China
}

\begin{abstract}
The laterodorsal thalamic nucleus (LD) is believed to play roles in learning and memory, especially spatial tasks. However, the molecular mechanism that underlies the cognitive process in the LD remains unclear and needs to be investigated. So far, there is plenty of evidence indicating that plasticity has been in some of the cortical or subcortical regions closely related to the LD, particularly stimulated by external learning tasks. Therefore, the present study aimed to test the hypothesis that similar effect exists in the LD. The transcription factor, CAMP-response element binding protein (CREB), works essentially in brain plasticity by tightly regulating the transcriptional level of memory-related target genes, and the increase of activated CREB (phosphorylated CREB, p-CREB) could facilitate memory consolidation. In this study, the siRNA against CREB was synthesized to down-regulate the CREB mRNA in the LD. After Morris water maze behavioral training, CREB siRNA rats exhibited a memory deficiency, significantly diverging from the control groups. In subsequent detection, the expression of p-CREB of these memory impairment rats attenuated. These results support the hypothesis that CREBmediated plasticity contributes to memory facilitation and consolidation in the LD.
\end{abstract}

Selective lesions experiments and behavioral tests have shown that the laterodorsal thalamic nucleus (LD) contributes to spatial learning and memory (van Groen et al. 2002; Edelstyn et al. 2006; Jankowski et al. 2013). However, lacking of further exploration, the molecular mechanisms governing plasticity involved in memory formation and enhancement still remain poorly understood. The present study sought to determine the plasticity in the LD and how it associates with learning and memory function of rodents.

As a part of the classical Papez circuit, the anterior thalamic nucleus (ATN) often generated more attention than LD. Lesions within this area led to deficits in multimodal learning tasks, including the amnesia (Harding et al. 2000), spatial (Warburton and Aggleton 1999; Aggleton and Nelson 2015), and reference and working memory impairments (Warburton et al. 1997; Frohardt et al. 2006). Connectionally, positionally, and structurally, the high similarity between the ATN and LD makes the latter comparable. The LD likewise is reciprocally connected to the anterior cingulate and retrosplenial cortices, and has input and output connections with the postsubiculum and subiculum (hippocampal formation) (Mathiasen et al. 2017). All these similar interactions within the ATN play a role in learning and memory process, primarily spatial tasks (Aggleton et al. 2010; Mitchell et al. 2018). In addition, a landmark study revealed the functional contributions of the $\mathrm{LD}$, conveying directional information through its afferents to the hippocampal formation (Mizumori and Williams 1993). Some LD neurons, also known as head direction cells, selectively discharged especially when an animal's head was set in a particular direction, regardless of the location. This effect would fade when animals in the darkness, indicating that LD neuron rely on visual clues to initialize the firing. Furthermore, HD cells are also found in the postsubiculum, retrosplenial cortex, (Chen et al. 1994) and ATN (Taube 1995). A recent research also indicated that, besides visual stimuli, LD and ATN neurons also responded to somatosensory and proprioceptive stimuli, and these sorts of stimuli collectively participated in the space orientation af-

\section{Corresponding author: binwang2008@126.com}

Article is online at http://www.learnmem.org/cgi/doi/10.1101/lm.049742. 119. ter integrating the internal and environmental information (Wijesinghe et al. 2015).

Long-term memory formation depends on the synthesis of new proteins, and CREB is a typical mediator involved in this process, modulating the transcription of genes associated with synaptic plasticity that underlie memory formation and enhancement (Silva et al. 1998; Scott et al. 2002). Immediate early genes (IEGs) family, whose promoters have cAMP-response elements, is one of the downstream target regulated by CREB, proved to implicate in neural plasticity during contextual fear conditioning (Baumgärtel et al. 2018) and spatial learning and memory along with CREB (Dumont et al. 2012). Not only that, the study also showed that the lesions in the ATN triggered the alteration of p-CREB and IEGs in distal sites such as hippocampal formation and retrosplenial cortex. These crossed connections from the cortex to the thalamic nuclei may be regarded as the neural basis of synchronal interregional activation contributing to spatial tasks processing (Sherman 2017), thus, we are able to add the LD to the range of our interest to determine the similar effect in this area.

\section{SiRNA against CREB suppresses CREB mRNA expression in the LD of rats}

CREB was down-regulated by stereotaxically injecting siRNA into the LD. The control rats were injected with scrambled siRNA (siScr) or liposome vehicles (LIPO). The injection site is shown in the stereotaxic coordinate diagram (Paxinos 2013) in Figure $1 \mathrm{~A}$. The animals were sacrificed 48,72 , and $96 \mathrm{~h}$, respectively, after the injection manipulation, and reverse transcription polymerase chain reaction (RT-PCR) was performed to detect the CREB mRNA of these three timing groups. Figure 1B,C shows the RT-PCR result. Statistical analysis of the three groups was tested using the one-way ANOVA test. There was an overall significant

\footnotetext{
(C) 2019 Xiang et al. This article is distributed exclusively by Cold Spring Harbor Laboratory Press for the first 12 months after the full-issue publication date (see http://learnmem.cshlp.org/site/misc/terms.xhtml). After 12 months, it is available under a Creative Commons License (AttributionNonCommercial 4.0 International), as described at http://creativecommons. org/licenses/by-nc/4.0/.
} 
A

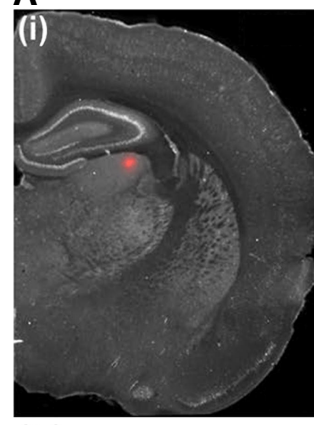

(iv)

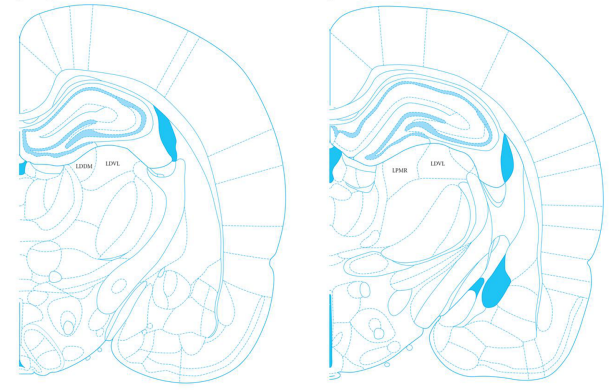

C

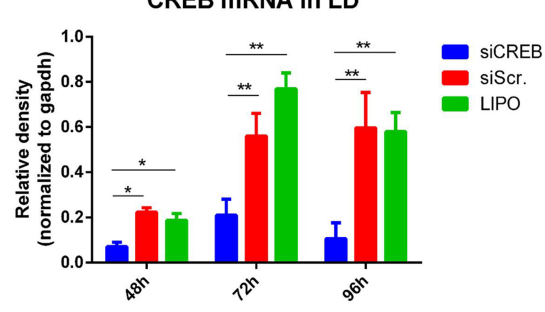

Figure 1. The injection site of the siRNA. Taking the bregma as the coordinate origin, the injection site was: Anterior/Posterior: $-2.5 \mathrm{~mm}$; Medial/Lateral: $\pm 2.4 \mathrm{~mm}$; Dorsal/Ventral: $-6.2 \mathrm{~mm}$. ( $A$ ) Verification of the injection sites by a fluorescent tracer. $(A \mathrm{i})$ shows the largest expand part of the $\mathrm{LD}$ at the coronal plane. (Aii) shows the most caudal portion of the LD at the coronal plane, (iii) and (iv) are schematic diagrams of $(A \mathrm{i})$ and $(A \mathrm{ii})$, respectively. $(B, C)$ PCR representative images and statistical analysis of the relative CREB mRNA level in each group. Lane 1: siCREB, lane 2: siScr, lane 3: LIPO. Error bars represent SD and significant differences are indicated by $\left(^{*}\right) P<0.05,\left({ }^{* *}\right) P<0.01 . N=9$. the spatial acquisition of siCREB group was also regular like any other groups $(P>0.05$, Parameter Estimates, Repeated Measures). Probe trial, a retention phase, which removed the platform in the tank was administered on day 4 . Referring to proposition of MWM memory modeling standards (Morris 1984; Vorhees and Williams 2006), the number of original platform crossings, escape latency (of probe trial), and the platform quadrant occupation time were used to assess the spatial memory consolidation, and the results are shown in Table 1. On the basis of standard control, control groups have successfully established spatial memory modeling, while siCREB has not.

Data analysis indicated significant differences in escape latency and crossing times (EL: $F=8.764, P<0.001$; Crossings: $F=9.513, \quad P=0.001$. one-way ANOVA). Post hoc test (Bonferroni) showed that siScr, LIPO and wild-type groups made considerably more times crossing the platform and had less escape latency than siCREB group (EL: SiCREB vs. siScr, $P=$ 0.025 ; siCREB vs. LIPO, $P<0.001$; siCREB vs. wild-type, $P=0.001$. Crossings: siCREB vs. siScr, $P=0.007$; siCREB vs. LIPO, $P=$ 0.003 ; siCREB vs. wild-type, $P=0.001$. Fig. 2C,D). Interestingly, differing from results above, analysis indicated the difference about platform quadrant occupation time was statistically nonsignificant

difference among the group. ( $48 \mathrm{~h}: F=17.122, P=0.003 ; 72 \mathrm{~h}: F=$ $34.350, P=0.001 ; 96 \mathrm{~h}: F=19.156, P=0.002$, Fig. 1C). Post hoc comparisons (Bonferroni) showed a significant decrease in the siCREB group compared to the control groups (48 h: siCREB vs. siScr, $P<0.05$, siCREB vs. LIPO, $P<0.05 ; 72$ h: siCREB vs. siScr, $P$ $<0.01$, siCREB vs. LIPO, $P<0.01 ; 96$ h: siCREB vs. siScr, $P<0.01$, siCREB vs. $L I P O, P<0.01)$. This verifies that the CREB was silenced by the siRNA.

\section{SiCREB rats exhibit memory impairment in Morris water maze, while acquisition stays intact}

Next, Morris water maze behavioral testing was conducted in injected (siCREB, siScr, LIPO) rats and wild-type rats. There are four different shaped distal clues placed around the tank to help rats to navigate their path toward the platform (Position: N, S, E, and $\mathrm{W})$. Besides, for avoiding the situation that rats learn a specific order in their movement to find the platform, we used different start positions that are approximate in length from the platform in each day's trial (Position: N, W, NW, and NE). The platform is located at center of the SE quadrant. All the rest procedures are implemented by standard MWM process of spatial version.

During place navigation (Fig. 2A, day1-day3) which serves as an acquisition phase, rats relied on various spatial strategies to locate the platform, followed by the formation of a cognitive spatial map. The escape latency, the time cost before the rats first landing on the platform, is used to assess the spatial ability. With the increase of training days, all groups showed a gradual learning process, and analysis of escape latency revealed a significant decrease over time in the acquisition phase $(F=140.757, P<0.05$, Tests of Within-Subjects Effects, Repeated Measures, Fig. 2B). Note that
$(F=1.947, P=0.163$. one-way ANOVA. Fig. $2 \mathrm{E})$, and we will discuss this situation below.

\section{The expression of $p$-CREB in rats with memory impairment is significantly decreased}

In many research studies that concentrated on other memoryrelated brain areas, like hippocampal formation or ATN, the level of phosphorylated CREB (p-CREB) would elevate after memory training, on the other hand, its decrease would impair spatial memory (Li et al. 2017; Yu et al. 2017). To examine this alteration whether it also exists in the LD, western blot and immunofluorescence were performed to measure p-CREB levels after behavioral testing. In addition, we set another untrained naive group, which all rats within it were put into tank to swim (the time of their swim was determined by the average of escape latency of the trained groups), but without the platform from the onset. The significant difference between WT and NG indicated that MWM training elevated the expression of p-CREB in the $\mathrm{LD}$, as showed in Figure $3 \mathrm{~A}$, B. A one-way ANOVA indicated that p-CREB levels among four trained groups were significantly different $(\mathrm{F}=17.666, P<0.001$, Fig. 3C,D). Post hoc test (Bonferroni) confirmed a significant decrease in siCREB group compared with control groups (siScr, $P<0.001$; LIPO, $P<0.001$; wt, $P<0.001$ ), and there were no statistical differences between control groups (siScr vs. LIPO , $P=1.000$; siScr vs. Wt, $P=0.573$; LIPO vs. Wt, $P=1.000$ ).

Immunofluorescence staining showed the distribution of p-CREB positive cells in the LD. As a nuclear protein, p-CREB is only expressed in the cell nucleus, and its immunoreactivity was determined by counting positive cells. As shown in Figure $3 \mathrm{~F}$, a one-way ANOVA indicated significant differences in p-CREB 
A

Day1
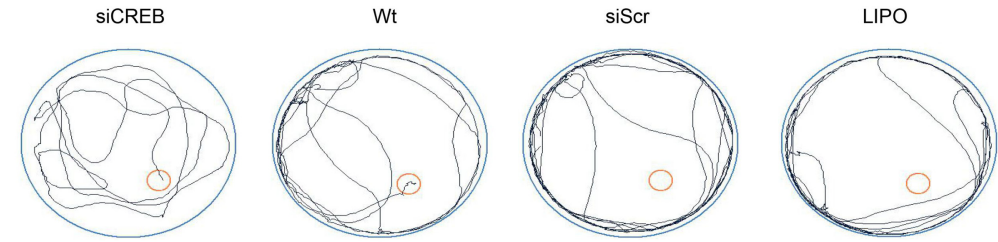

Day2
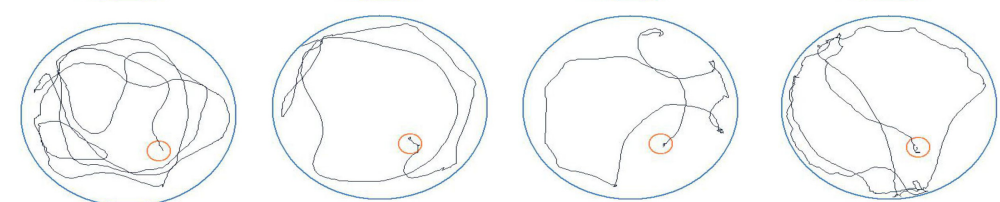

Day3
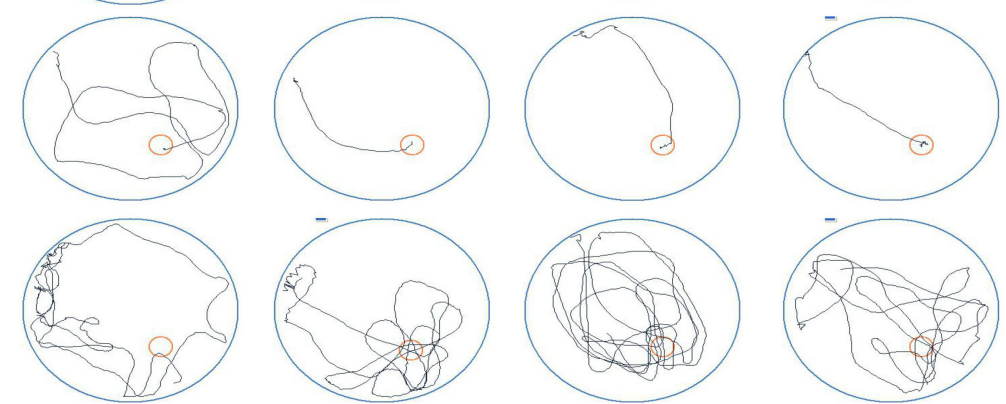

Probe

Trial

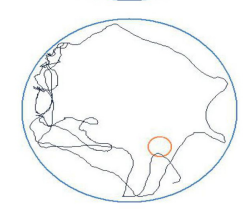

B

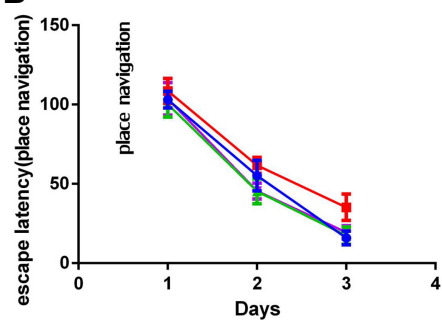

D

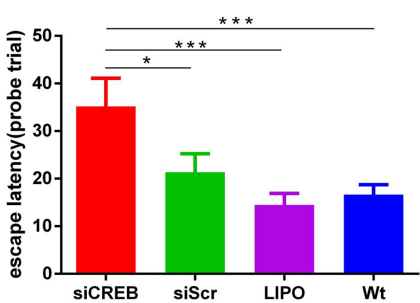

$\rightarrow W t$

- siCREB

- siScr

* LIPO

$c$

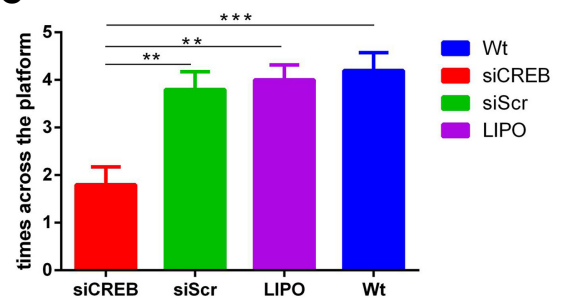

E

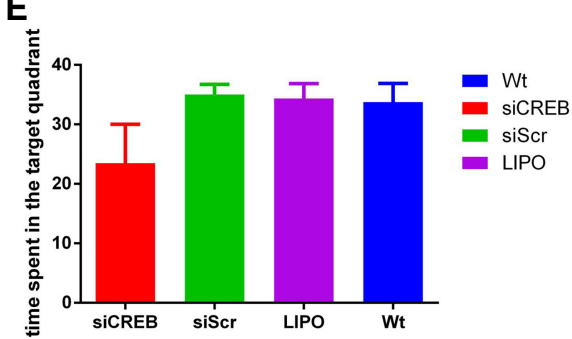

Figure 2. (A) The tracks of rats during place navigation and probe trial. From day 1 to day 3 , all groups experienced a gradual learning process. In day 4 of probe trial, siCREB group obviously differed from siScr, LIPO, and wild-type rats, showing less platform crossings and more indistinct tendency toward the platform. (B) Analysis of escape latency in place navigation. All groups of rats exhibited spatial learning. ( $C-E$ ) Comparison of escape latency in probe trial, of the number of platform crossings and of the time spent in the platform quadrant. Error bars represent SD and significant differences are indicated by (*) $P<0.05,\left({ }^{* *}\right) P<0.01,\left(^{* * *}\right) P<0.001 . N=10$ rats in each group.

positive cells among four groups $(F=35.434, P<0.001)$. Positive cells were strongly expressed in the control groups, and almost absent from the siCREB and NG groups (siScr vs. siCREB, $P<0.001$;

LIPO vs. SiCREB, $P<0.001$; WT vs siCREB, $P=0.001$. siScr vs. NG, $P<0.001$; LIPO vs. NG, $P<0.001$; WT vs. NG, $P=$ 0.006 . Post hoc comparison). The expression among control groups were not statistically significant (siScr vs. LIPO,$P=$ 1.000 ; siScr vs. WT, $P=0.372$; LIPO vs. WT, $P=0.288$ ). The results of immunofluorescence also support those of the western blot.

CREB has been widely studied as a transcription factor involved in the formation and consolidation of memory, especially long-term memory. Bilateral down-regulation of CREB mRNA in the LD lead to an underperformance in behavioral testing for spatial memory retention, subsequently, the level of activated CREB in memory impairment group was also much lower than that in control groups. Thus we identified a direct association between spatial memory and p-CREB level in the LD, indicating the plasticity in the LD also contributes to spatial memory consolidation.

In behavioral testing, the probe trial, we came across interesting results. The insignificance between treated group and control groups appearing in the behavioral statistics about platform quadrant occupation time was an issue worth attention, as it was not consistent with our other results. To exclude an accident, we increased the number of cases and finally found it remained the same. We found that siCREB group data always had a greater discretization, which is represented in Figure 2E by standard deviation, than that of control groups, even before the increasing of cases. That means occupation time varied widely from rat to rat within siCREB group. Visual inspection of movements of siCREB rats during the probe trial also allied the digits. SiCREB rats whose occupation time was low did not show any specificity for the platform or corresponding quadrant, and their trajectories were "randomly" distributed among four quadrants. Contrastively, high-occupation rats spent more time as well as traveled much longer paths in the platform quadrant. But, crucially, high-occupation rats did not show more accurate sense about the specific location of the platform relative to low-occupation rats, as if they solely knew the general area where the platform is. This could explain why differences were so apparent at the number of

Table 1. Escape latency, platform quadrant occupation time, and original platform crossing times are main reference indexes in Morris water maze. Groups that meet the requirements of standard control could be regarded as the ones with intact memory functions

\begin{tabular}{lccccc}
\hline & siCREB & siScr & LIPO & WT & Standards control \\
\hline Escape latency & $35.1778 \pm 12.22812$ & $21.3556 \pm 9.84341$ & $14.4667 \pm 6.55763$ & $16.5400 \pm 8.58347$ & $\leq 30$ \\
Occupation time & $23.4800 \pm 14.59579$ & $35.0200 \pm 3.82714$ & $34.3600 \pm 5.57387$ & $33.7600 \pm 7.00914$ & $\geq 30$ \\
Crossing times & $1.8000 \pm 0.83666$ & $3.8000 \pm 0.83666$ & $4.0000 \pm 0.70711$ & $4.2000 \pm 0.83666$ & $\geq 2$ \\
\hline
\end{tabular}


A
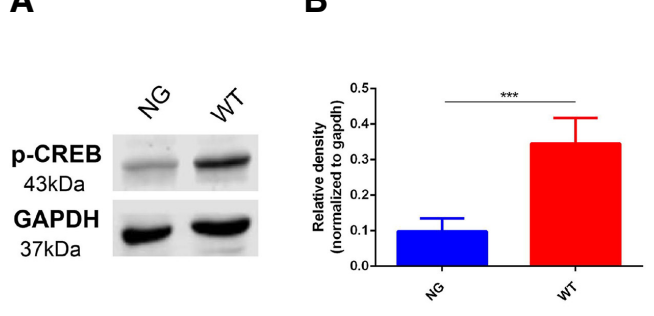

D

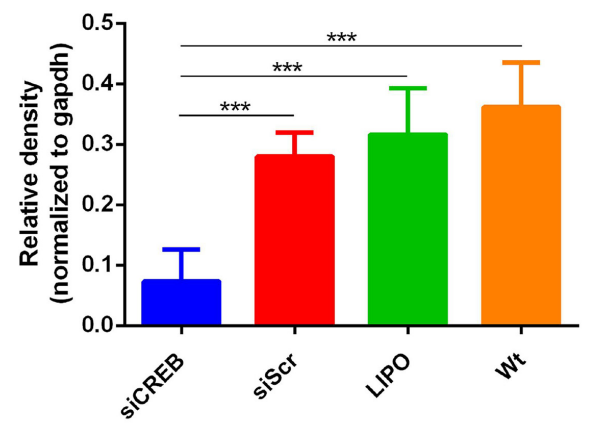

E

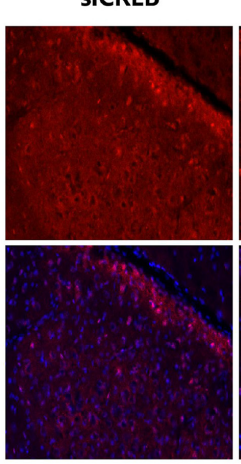

B
siScr

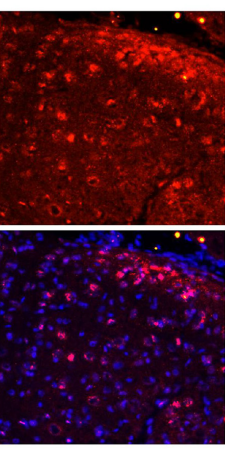

C

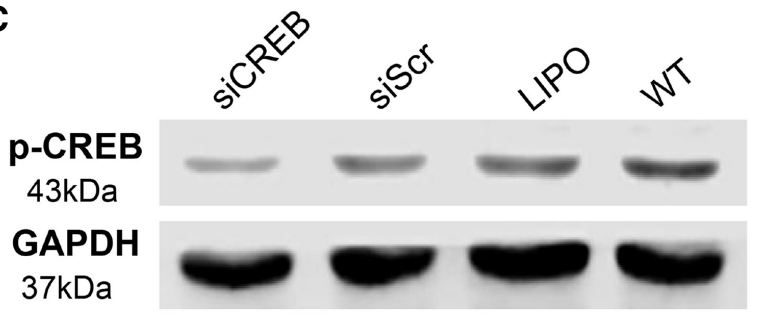

$\mathbf{F}$

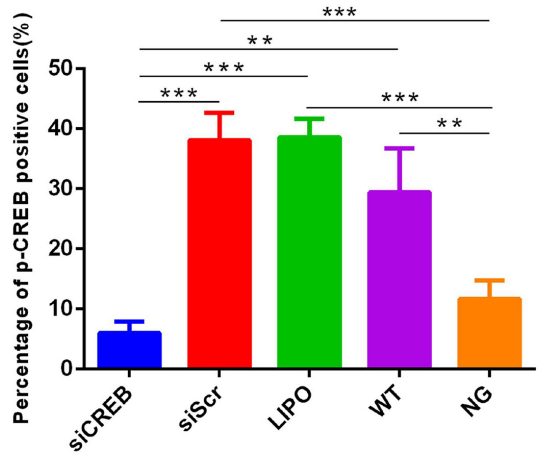

WT

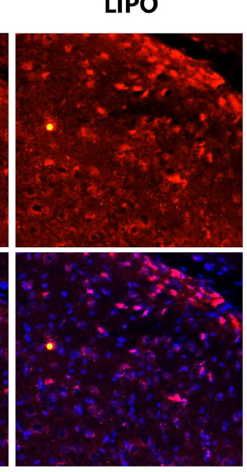

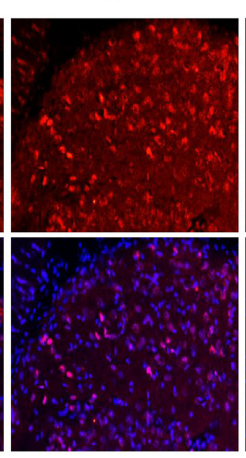

NG

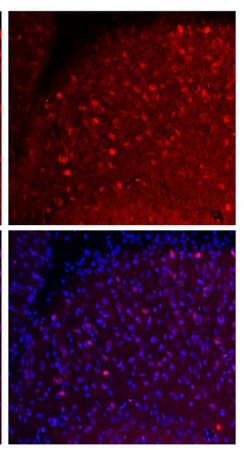

Figure 3. $(A-D)$ Western blot representative images and statistic analysis of protein level of $p-C R E B$. (E) Immunofluorescence representative images. Images of WT and NG were collected from ventrolateral subregion of the LD (LDVL) in the left hemispheres, and images of siCREB, siScr, and LIPO groups were collected from LDVL in the right hemisphere. Positive p-CREB cells were expressed in the nucleus, presenting approximately pink after merging with DAPI staining. (D) The percentage of positive $p$-CREB cells of the total number of cells, quantified and analyzed by Image Pro Plus 7.0 (Media Cybernetics, Inc.). Error bars represent SD and significant differences are indicated by $\left(^{*}\right) P<0.05,\left({ }^{* *}\right) P<0.01,\left({ }^{* *}\right) P<0.001 . N=5-6$ rats in each group.

platform crossings and escape latency (of probe trial). In addition, there is other research supporting this view-for rats that have intact memory function, they may underperform in quadrant preference, when not coming along with higher quality of distal cues (Rogers et al. 2017). In our study, the quality of distal cues is quite moderate, four difform cards which are flat and plain, while the high quality means an array of salient cues. So it also could bring on a narrower gap between siCREB and control group in quadrant preference.

Some animal experiments have revealed the characteristics of LD and the memory system that it may reside in. In some paired-associate learning or spatial processing tasks, when lesions concentrated solely on the LD or other adjacent nuclei, rats tended not to show conspicuous deficits in memory function. Once lesion range extended to multiple nuclei, the damage effect would be much more severe, and would not be able to recover with training sessions increased (Dillingham et al. 2015; Leong et al. 2016). In combination with the result of the present study, the interference of CREB signaling pathway in the LD did not severely restrict the spatial acquisition, we tend to think each nucleus does not work individually on acquisition of an ability to learn or memorize. In brief, our work provides evidence for the presence of memory-related signaling pathway in the LD, which may further explore the role of the LD in the synergy among various nuclei of the thalamus during learning and memory.

\section{References}

Aggleton JP, Nelson AJ. 2015. Why do lesions in the rodent anterior thalamic nuclei cause such severe spatial deficits? Neurosci Biobehav Rev 54: 131-144. doi:10.1016/j.neubiorev.2014.08.013

Aggleton JP, O’Mara SM, Vann SD, Wright NF, Tsanov M, Erichsen JT. 2010. Hippocampal-anterior thalamic pathways for memory: uncovering a network of direct and indirect actions. Eur J Neurosci 31: 2292-2307. doi:10.1111/j.1460-9568.2010.07251.x

Baumgärtel K, Green A, Hornberger D, Lapira J, Rex C, Wheeler DG, Peters M. 2018. PDE4D regulates spine plasticity and memory in the retrosplenial cortex. Sci Rep 8: 3895. doi:10.1038/s41598-018-22193-0

Chen LL, Lin LH, Green EJ, Barnes CA, McNaughton BL. 1994. Head-direction cells in the rat posterior cortex. I. Anatomical 
distribution and behavioral modulation. Exp Brain Res 101: 8-23. doi:10 $.1007 /$ BF00243212

Dillingham CM, Erichsen JT, O'Mara SM, Aggleton JP, Vann SD. 2015. Fornical and nonfornical projections from the rat hippocampal formation to the anterior thalamic nuclei. Hippocampus 25: 977-992. doi:10.1002/hipo.22421

Dumont JR, Amin E, Poirier GL, Albasser MM, Aggleton JP. 2012. Anterior thalamic nuclei lesions in rats disrupt markers of neural plasticity in distal limbic brain regions. Neuroscience 224: 81-101. doi:10.1016/j .neuroscience.2012.08.027

Edelstyn NM, Hunter B, Ellis SJ. 2006. Bilateral dorsolateral thalamic lesions disrupts conscious recollection. Neuropsychologia 44: 931-938. doi:10 .1016/j.neuropsychologia.2005.08.012

Frohardt RJ, Bassett JP, Taube JS. 2006. Path integration and lesions within the head direction cell circuit: comparison between the roles of the anterodorsal thalamus and dorsal tegmental nucleus. Behav Neurosci 120: $135-149$. doi: $10.1037 / 0735-7044.120 .1 .135$

Harding A, Halliday G, Caine D, Kril J. 2000. Degeneration of anterior thalamic nuclei differentiates alcoholics with amnesia. Brain 123: 141154. doi:10.1093/brain/123.1.141

Jankowski MM, Ronnqvist KC, Tsanov M, Vann SD, Wright NF, Erichsen JT, Aggleton JP, O'Mara SM. 2013. The anterior thalamus provides a subcortical circuit supporting memory and spatial navigation. Front Syst Neurosci 7: 45. doi:10.3389/fnsys.2013.00045

Leong AT, Chan RW, Gao PP, Chan YS, Tsia KK, Yung WH, Wu EX. 2016. Long-range projections coordinate distributed brain-wide neural activity with a specific spatiotemporal profile. Proc Natl Acad Sci 113: E8306-E8315. doi:10.1073/pnas.1616361113

Li X, Guo C, Li Y, Li L, Wang Y, Zhang Y, Li Y, Chen Y, Liu W, Gao L. 2017. Ketamine administered pregnant rats impair learning and memory in offspring via the CREB pathway. Oncotarget 8: 32433-32449. doi:10 .18632 /oncotarget.15405

Mathiasen ML, Dillingham CM, Kinnavane L, Powell AL, Aggleton JP. 2017. Asymmetric cross-hemispheric connections link the rat anterior thalamic nuclei with the cortex and hippocampal formation. Neuroscience 349: 128-143. doi:10.1016/j.neuroscience.2017.02.026

Mitchell AS, Czajkowski R, Zhang N, Jeffery K, Nelson AJD. 2018. Retrosplenial cortex and its role in spatial cognition. Brain Neurosci Adv 2: 2398212818757098 . doi:10.1177/2398212818757098

Mizumori SJ, Williams JD. 1993. Directionally selective mnemonic properties of neurons in the lateral dorsal nucleus of the thalamus of rats. J Neurosci 13: 4015-4028. doi:10.1523/JNEUROSCI.13-09-04015 .1993
Morris R. 1984. Developments of a water-maze procedure for studying spatial learning in the rat. J Neurosci Methods 11: 47-60. doi:10.1016/ 0165-0270(84)90007-4

Paxinos G. 2013. The rat brain in stereotaxic coordinates, 7 th ed. Academic Press.

Rogers J, Churilov L, Hannan AJ, Renoir T. 2017. Search strategy selection in the Morris water maze indicates allocentric map formation during learning that underpins spatial memory formation. Neurobiol Learn Mem 139: 37-49. doi:10.1016/j.nlm.2016.12.007

Scott R, Bourtchuladze R, Gossweiler S, Dubnau J, Tully T. 2002. CREB and the discovery of cognitive enhancers. J Mol Neurosci 19: 171-177. doi:10 $.1007 / \mathrm{s} 12031-002-0029-\mathrm{z}$

Sherman SM. 2017. Functioning of circuits connecting thalamus and cortex. Compr Physiol 7: 713-739. doi:10.1002/cphy.c160032

Silva AJ, Kogan JH, Frankland PW, Kida S. 1998. CREB and memory. Annu Rev Neurosci 21: 127-148. doi:10.1146/annurev.neuro.21.1.127

Taube JS. 1995. Head direction cells recorded in the anterior thalamic nuclei of freely moving rats. J Neurosci 15: 70-86. doi:10.1523/JNEUROSCI .15-01-00070.1995

van Groen T, Kadish I, Wyss JM. 2002. The role of the laterodorsal nucleus of the thalamus in spatial learning and memory in the rat. Behav Brain Res 136: 329-337. doi:10.1016/S0166-4328(02)00199-7

Vorhees CV, Williams MT. 2006. Morris water maze: procedures for assessing spatial and related forms of learning and memory. Nat Protoc $\mathbf{1}$ : 848-858. doi:10.1038/nprot.2006.116

Warburton EC, Aggleton JP. 1999. Differential deficits in the Morris water maze following cytotoxic lesions of the anterior thalamus and fornix transection. Behav Brain Res 98: 27-38. doi:10.1016/S0166-4328(98) 00047-3

Warburton EC, Baird AL, Aggleton JP. 1997. Assessing the magnitude of the allocentric spatial deficit associated with complete loss of the anterior thalamic nuclei in rats. Behav Brain Res 87: 223-232. doi:10.1016/ S0166-4328(97)02285-7

Wijesinghe R, Protti DA, Camp AJ. 2015. Vestibular interactions in the thalamus. Front Neural Circuits 9: 79. doi:10.3389/fncir.2015.00079

Yu XW, Curlik DM, Oh MM, Yin JC, Disterhoft JF. 2017. CREB overexpression in dorsal CA1 ameliorates long-term memory deficits in aged rats. Elife 6: e19358. doi:10.7554/eLife.19358

Received March 17, 2019; accepted in revised form April 19, 2019. 


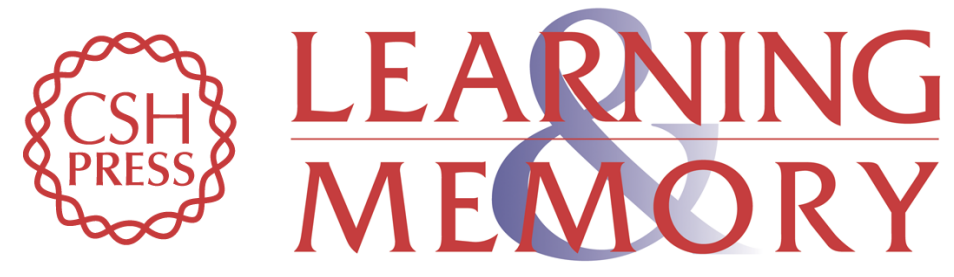

\section{CREB down-regulation in the laterodorsal thalamic nucleus deteriorates memory consolidation in rats}

Wenxi Xiang, Tingting Li, Tianhang Gao, et al.

Learn. Mem. 2019, 26:

Access the most recent version at doi:10.1101/lm.049742.119

References This article cites 27 articles, 3 of which can be accessed free at: http://learnmem.cshlp.org/content/26/6/182.full.html\#ref-list-1

Creative This article is distributed exclusively by Cold Spring Harbor Laboratory Press for the Commons License first 12 months after the full-issue publication date (see

http://learnmem.cshlp.org/site/misc/terms.xhtml). After 12 months, it is available under a Creative Commons License (Attribution-NonCommercial 4.0 International), as described at http://creativecommons.org/licenses/by-nc/4.0/.

Email Alerting Receive free email alerts when new articles cite this article - sign up in the box at the Service top right corner of the article or click here. 